\title{
Impact of the Patterns of Retropharyngeal Lymph Node Metastasis on the Clinical Target Volume for the Radiotherapy Treatment of Nasopharyngeal Carcinoma
}

\author{
Lihua Tong, Wen Yang, Xingxi Pan* \\ Department of Oncology, Nanhai Hospital Affiliated to Southern Medical University, Foshan, China \\ Email address: \\ pxx6797@163.com (Xingxi Pan) \\ ${ }^{*}$ Corresponding author
}

To cite this article:

Lihua Tong, Wen Yang, Xingxi Pan. Impact of the Patterns of Retropharyngeal Lymph Node Metastasis on the Clinical Target Volume for the Radiotherapy Treatment of Nasopharyngeal Carcinoma. International Journal of Clinical Oncology and Cancer Research.

Vol. 4, No. 4, 2019, pp. 34-37. doi: 10.11648/j.ijcocr.20190404.11

Received: June 3, 2019; Accepted: July 8, 2019; Published: October 31, 2019

\begin{abstract}
Objective: The aim of this study was to explore the patterns of retropharyngeal lymph node (RLN) metastasis in nasopharyngeal carcinoma (NPC) and their impact on the clinical target volume (CTV) delineation in radiotherapy. Methods: A total of 190 patients with untreated, nonmetastatic NPC received MRI scans of the nasopharynx and neck before treatment. The imaging characteristics of RLN metastasis and their relationships with the staging system were analysed. Results: A total of 121 patients developed RLN metastasis. The incidence rate of RLN metastasis was $63.7 \%$. The RLN metastases of the 121 patients were distributed evenly in the lateral group, and no lymph node metastasis was observed in the medial group. Among the 121 patients, there were 66 cases of unilateral metastasis $(54.5 \%)$ and 55 cases of bilateral metastasis $(45.5 \%)$. The central position of the lymph nodes was mainly distributed in the $\mathrm{C} 1$ vertebra region. The differences in the RLN metastasis rate between different $\mathrm{T}$ stages, $\mathrm{N}$ stages and clinical stages were statistically significant (all $\mathrm{P}<0.01$ ). Conclusion: Medial RLN metastasis is rarely observed in NPC and therefore does not require routine prophylactic irradiation with intensity-modulated radiation therapy (IMRT). This is an important issue for future research.
\end{abstract}

Keywords: Nasopharyngeal Carcinoma (NPC), Retropharyngeal Lymph Node (RLN), Radiation Therapy, Clinical Target Volume (CTV)

\section{Introduction}

Although Nasopharyngeal carcinoma (NPC) is rare in many parts of the world, it is endemic in certain regions, especially in Southeast Asia. Radiotherapy is the mainstay treatment for NPC. In recent years, IMRT has gradually replaced conventional radiation therapy as the main radiotherapy technology used to treat NPC. The prerequisite for the development and application of IMRT is the correct delineation of the target areas. This allows tumour tissue to receive an accurate dose of radiation while protecting normal tissue. Theoretically, reducing the clinical target area can reduce the radiation volume, which can relieve adverse radiotherapy reactions [1-2]. To understand the patterns of lymph node metastasis, it is helpful to optimize and standardize the CTV delineation of the neck for NPC.
Previous studies have shown that avoiding exposure of the medial area of the RLN can reduce the dose of radiation received by the pharyngeal constrictor and posterior mucosa of the pharynx, thereby reducing the incidence of mucositis and dysphagia [3-4]. Therefore, in this study, we explored the patterns of RLN metastasis in NPC in 183 patients to provide a basis for the optimization of the CTV delineation of the neck for the treatment of NPC with IMRT.

\section{Methods and Materials}

\subsection{Patient Characteristics}

A total of 190 patients with newly diagnosed, biopsy-confirmed, nonmetastatic NPC treated at the Department of Oncology, Nanhai Hospital Affiliated with the Southern Medical University with IMRT between January 
2009 and December 2017 were retrospectively reviewed. The cohort included 141 males and 49 females, with a median age of 46 years (range, 15-81 years of age). These patients were assessed by staging with the 8th Edition of the UICC/AJCC Staging System for NPC. All patients were required to undergo a detailed physical examination, haematology and biochemistry profiles, nasopharyngeal fibrescope examination, chest radiograph, abdominal ultrasound, whole-body bone scan, and magnetic resonance imaging (MRI) of the nasopharynx and neck. The patient clinical characteristics are shown in Table 1.

Table 1. Characteristics of study patients.

\begin{tabular}{ll}
\hline Characteristics & N (\%) \\
\hline Age (y) & \\
Median & 46 \\
Range & $15-81$ \\
Gender & \\
Male & $141(74.21 \%)$ \\
Female & $49(25.79 \%)$ \\
Histology & \\
WHO Type I & $1(0.53 \%)$ \\
WHO Type II & $2(1.05 \%)$ \\
WHO Type II & $187(98.42 \%)$ \\
T classification & \\
T1 & $23(12.11 \%)$ \\
T2 & $93(48.95 \%)$ \\
T3 & $39(20.53 \%)$ \\
T4 & $35(18.42 \%)$ \\
N classification & \\
N0 & $6(3.16 \%)$ \\
N1 & $45(23.68 \%)$ \\
N2 & $86(45.26 \%)$ \\
N3 & $53(27.89 \%)$ \\
Stage & \\
I & $5(2.63 \%)$ \\
II & $26(13.68 \%)$ \\
III & $76(40.00 \%)$ \\
IVa & $83(43.68 \%)$ \\
\hline
\end{tabular}

\subsection{Imaging Protocol}

MRI was performed with a 1.5-T system (GE Signa Infinity Echospeed Plus 1.5T). The region from the suprasellar cistern to the inferior margin of the sternal end of the clavicle was examined with a head and neck combined coil. T1-weighted fast spin-echo images of the axial, coronal, and sagittal planes and T2-weighted fast spin-echo images of the axial plane were obtained before injection of the contrast material. After intravenous injection of the contrast material, T1-weighted axial and sagittal sequences and T1-weighted fat-suppressed coronal sequences were performed sequentially. All data were uploaded to the PACS system.

\subsection{Criteria for RLN Metastasis}

The MRI diagnostic criteria for RLN metastasis included the following [5-7]: (a) any individual lateral RLN with a minimal axial diameter in the largest plane of at least $5 \mathrm{~mm}$; (b) any node seen in the medial retropharyngeal group; and (c) lymph nodes of any size with central necrosis or extracapsular spread.

\subsection{MRI Analysis and Clinical Assessment}

MRI images were analysed by two senior radiotherapy physicians. The imaging features for RLN metastasis were analysed. The minimal and maximal axial diameters in the largest plane, location (central position, cranial border and caudal border), number of metastases, and presence of unilateral or bilateral metastases were recorded. All patients were staged according to the 8th Edition of the UICC/AJCC Staging System for NPC, in which RLN metastasis is classified as N1 stage. In the event of a disagreement, the stage was decided by the research group.

\subsection{Statistical Analysis}

SPSS 20.0 software was used for statistical analysis. The measurement data were expressed as the mean \pm standard deviation. The relationship between RLN metastasis and clinical staging was analysed by chi-square test. P values $<0.05$ were considered statistically significant in all analyses.

\section{Results}

\subsection{Patterns and Characteristics of RLN Metastasis}

A total of 121 patients from the whole group had RLN metastasis, and the rate of RLN metastasis was $63.7 \%$ (121/190). The RLN metastasis of the 121 patients was distributed evenly in the lateral group and no lymph node metastasis was seen in the medial group. Among the patients with RLN metastasis, there were 66 patients with unilateral metastases (54.5\%) and 55 patients with bilateral metastases (45.5\%). There were 219 RLN metastases, including 53 patients with 1 metastasis, 42 patients with 2, 22 patients with 3 and 4 patients with 4 . The metastatic lymph nodes were distributed horizontally with 1-3 lymph nodes involved and longitudinally with 1-4 lymph nodes involved. The mean values of the minimal and maximal axial diameters in the largest plane of the positive nodes were $14.54 \pm 6.74 \mathrm{~mm}$ and $10.25 \pm 4.35 \mathrm{~mm}$, respectively. The locations of the RLN metastases in NPC patients are shown in Table 2.

Table 2. Location of RLN metastases in 121 patients with NPC.

\begin{tabular}{|c|c|c|c|}
\hline & Central location of RLN & Cranial border of RLN & Caudal border of RLN \\
\hline Occipital bone & $13(10.74 \%)$ & $57(47.11 \%)$ & $12(9.92 \%)$ \\
\hline $\mathrm{C} 1$ & $79(65.29 \%)$ & $46(38.02 \%)$ & $58(47.93 \%)$ \\
\hline $\mathrm{C} 2$ & $29(23.97 \%)$ & $18(14.88 \%)$ & $49(40.50 \%)$ \\
\hline $\mathrm{C} 3$ & $0(0.00 \%)$ & $0(0.00 \%)$ & $2(1.65 \%)$ \\
\hline
\end{tabular}




\subsection{Relationship Between RLN Metastasis and Disease Stage According to the 8th Edition of the UICC/AJCC Staging System for NPC}

According to staging with the 8th Edition of the UICC/AJCC Staging System for NPC, the differences in the RLN metastasis rates between different $\mathrm{T}$ stages, $\mathrm{N}$ stages and clinical stages were statistically significant (all $\mathrm{P}<0.01$ ). The results are shown in Table 3.

Table 3. Relationship between RLN metastasis and the disease stage according to the 8th Edition of the UICC/AJCC Staging System.

\begin{tabular}{lllll} 
& \multicolumn{2}{c}{ RLN metastasis } & \multirow{2}{*}{$\boldsymbol{\chi}^{2}$} & \multirow{2}{*}{ P } \\
\cline { 2 - 3 } & Yes & No & & \\
\hline T staging & & & & \\
T1 & $7(3.68 \%)$ & $16(8.42 \%)$ & & \\
T2 & $70(36.84 \%)$ & $23(12.11 \%)$ & & \\
T3 & $26(13.68 \%)$ & $13(6.84 \%)$ & & \\
T4 & $18(9.47 \%)$ & $17(8.95 \%)$ & & \\
N staging & & & & \\
N0 & $0(0.00 \%)$ & $6(3.16 \%)$ & & \\
N1 & $23(12.11 \%)$ & $22(11.58 \%)$ & & \\
N2 & $54(28.42 \%)$ & $32(16.84 \%)$ & & \\
N3 & $44(23.16 \%)$ & $9(4.74 \%)$ & & \\
Clinical staging & $0(0.00 \%)$ & $5(2.63 \%)$ & & \\
I & $12(6.32 \%)$ & $14(7.37 \%)$ & & \\
II & $50(26.32 \%)$ & $26(13.68 \%)$ & & \\
III & $59(31.05 \%)$ & $24(12.63 \%)$ & & \\
IV & & & & \\
\hline
\end{tabular}

\section{Discussion}

As the nasopharynx has a well-developed network of lymphatics, NPC has a higher incidence of cervical lymph node metastasis compared with other head and neck cancers. At present, the first echelon lymph node of NPC is still controversial and is reported to be either the RLNs or level II lymph nodes. Several reports have shown that both the RLNs and level II lymph nodes are first echelon nodes in NPC [5, 6, 8]. The MRI data in the present study demonstrated that $63.7 \%$ of 190 patients had retropharyngeal lymph node metastasis, which was consistent with the results reported by Yue et al. [9] and Ho et al. [10].

The RLNs lie within the retropharyngeal space, extending cranially from the upper edge of the first cervical vertebrae to the cranial edge of the body of the hyoid bone caudally [11]. This space is bounded laterally by the medial edge of the internal carotid artery, posteriorly by the longus capitis and longus colli muscles and anteriorly by the pharyngeal constrictor muscles. RLNs are classified into lateral and medial groups. The lateral group lies medial to the carotid arteries and lateral to the longus colli and capitis muscles, whereas the medial group is intercalated along lymphatics near the midline [3]. In this study, RLN metastasis is related to the $\mathrm{T}$ stage, $\mathrm{N}$ stage and clinical stage as assessed by the 8th Edition of the UICC/AJCC Staging System for NPC. All metastatic RLNs were found in the lateral group, whereas no metastases were found in the medial group in our study. Since medial group metastasis is rarely observed in NPC, any visible medial RLN was defined as a metastasis. In our review of the literature, we found no more than 10 cases have been reported [5-8].

The IMRT technique, which has gradually replaced conventional radiotherapy as the standard treatment modality for NPC, improves the efficacy and quality of life of patients with NPC. The target volume delineation of NPC for IMRT mainly referred to the RTOG 0025 and 0615 protocols in the past, which were largely based on previous experiences in conventional radiotherapy [12]. These protocols clearly defined the CTV as including the RLNs from the base of the skull to the cranial edge of the hyoid [13-15]. Several studies have shown that in almost all cases in which the RLNs are at risk, only the lateral RLNs are at substantial risk [2-4]. Consequently, some researchers have proposed selective delineation of the medial group [1].

A study by Lin et al. [16] reported the treatment results for 323 patients with NPC treated with reduced-volume IMRT. In their study, the CTV included the retropharyngeal nodal regions from the base of the skull to the cranial edge of the second cervical vertebra (C2). In a median follow-up of 30 months, the 3 -year local control, regional control, and OS rates were $95 \%$, $98 \%$, and $90 \%$, respectively. There was no regional failure in the unirradiated retropharyngeal nodal area. Their update demonstrated an excellent local control rate of $95 \%$ at 5 years, and the 5-year OS rate was $80 \%$ [12]. Yi et al. [17] reported their IMRT experience in 376 NPC patients; they delineated the CTV at $1.0 \mathrm{~cm}-1.5 \mathrm{~cm}$ below the GTV or the cranial edge of $\mathrm{C} 1$ if the tumour was confined to the parietal wall and excluded the medial RLNs to reduce the severe early or late radiation damage to the oropharynx. Wang et al.[4] showed that 2012 (75.1\%) of 3100 patients had RLN metastasis; only 6 metastases $(0.2 \%)$ were located in the medial group, and they were accompanied by lateral RLN and other node metastases. They have also reported results for IMRT excluding the medial RLNs below one third of the second cervical vertebra (C2) in NPC patients since 2005 and observed no regional failures in the medial group. Based on the above results, the 2010 Chinese Consensus Guidelines for NPC IMRT Planning suggested that the medial RLNs between the $\mathrm{C} 2$ level and the hyoid bone can be excluded from the CTV when treating NPC [18].

International guidelines for the delineation of the CTV for NPC treatment have issued and are aimed at providing clinicians with a practical reference on treatment principles in NPC [19]. In this guideline, only the lateral group needs prophylactic irradiation to avoid too much of the pharyngeal constrictors being unnecessarily irradiated. Our results also showed that the medial group was rarely involved, and we suggested that the medial RLNs should not be defined as targets. However, further work is needed to fully understand the impact of medial RLN sparing on survival of NPC patients.

\section{Conclusion}

In this study, metastasis to the medial RLNs is rarely observed in NPC patients; therefore, the medial RLNs do not 
require routine prophylactic irradiation by IMRT. However, this study has certain limitations. First, this was a retrospective study. Second, the sample size was small. Further studies need to be carried out to evaluate whether medial RLN sparing could improve the quality of life of NPC patients without compromising their survival rate.

\section{Conflict of Interest}

The authors declare that they have no competing interests.

\section{Acknowledgements}

This work was supported by grants from the Medical Science Technology Project of Foshan City, China (No: 2017AB000422).

\section{References}

[1] Dirix P, Nuyts S. Evidence-based organ-sparing radiotherapy in head and neck cancer [J]. Lancet Oncology, 2010, 11 (1): 85-91.

[2] Feng F Y, Kim H M, Lyden T H, et al. Intensity-modulated chemoradiotherapy aiming to reduce dysphagia in patients with oropharyngeal cancer: clinical and functional results. [J]. Journal of Clinical Oncology Official Journal of the American Society of Clinical Oncology, 2010, 28 (16): 2732.

[3] Feng F Y, Kim H M, Lyden T H, et al. Intensity-modulated radiotherapy of head and neck cancer aiming to reduce dysphagia: early dose-effect relationships for the swallowing structures. [J]. Int J Radiat Oncol Biol Phys, 2007, 68 (5): 1289-1298.

[4] Wang X S, Yan C, Hu C S, et al. Study of the medial group retropharyngeal node metastasis from nasopharyngeal carcinoma based on 3100 newly diagnosed cases [J]. Oral Oncol, 2014, 50 (11): 1109-1113.

[5] Tang L, Mao Y, Liu L, et al. The volume to be irradiated during selective neck irradiation in nasopharyngeal carcinoma: analysis of the spread patterns in lymph nodes by magnetic resonance imaging. [J]. Cancer, 2009, 115 (3): 680-688.

[6] Wang X S, Hu C S, Ying H M, et al. Patterns of retropharyngeal node metastasis in nasopharyngeal carcinoma. [J]. International Journal of Radiation Oncology Biology Physics, 2009, 73 (1): 194-201.

[7] Liu L Z, Zhang G Y, Xie C M, et al. Magnetic resonance imaging of retropharyngeal lymph node metastasis in nasopharyngeal carcinoma: patterns of spread [J]. International Journal of Radiation Oncology Biology Physics, 2006, 66 (3): 721-730.
[8] Ng S H, Chang J T, Chan S C, et al. Nodal metastases of nasopharyngeal carcinoma: patterns of disease on MRI and FDG PET [J]. European Journal of Nuclear Medicine \& Molecular Imaging, 2004, 31 (8): 1073-1080.

[9] Yue D, Xu Y F, Zhang F, et al. Is replacement of the supraclavicular fossa with the lower level classification based on magnetic resonance imaging beneficial in nasopharyngeal carcinoma? [J]. Radiotherapy \& Oncology, 2014, 113 (1): 108-114.

[10] Ho F C, Tham I W, Earnest A, et al. Patterns of regional lymph node metastasis of nasopharyngeal carcinoma: A meta-analysis of clinical evidence [J]. Bmc Cancer, 2012, 12 (1): 98.

[11] Grégoire V, Ang K, Budach W, et al. Delineation of the neck node levels for head and neck tumors: A 2013 update. DAHANCA, EORTC, HKNPCSG, NCIC CTG, NCRI, RTOG, TROG consensus guidelines [J]. Radiotherapy \& Oncology Journal of the European Society for Therapeutic Radiology \& Oncology, 2014, 110 (1): 172-181.

[12] Lin S, Pan J, Han L, et al. Update report of nasopharyngeal carcinoma treated with reduced-volume intensity-modulated radiation therapy and hypothesis of the optimal margin [J]. Radiotherapy \& Oncology, 2014, 110 (3): 385-389.

[13] Lee N Y, Zhang Q, Pfister D G, et al. Addition of bevacizumab to standard chemoradiation for locoregionally advanced nasopharyngeal carcinoma (RTOG 0615): a phase 2 multi-institutional trial. [J]. Lancet Oncology, 2012, 13 (2): 172-180.

[14] Radiation Therapy Oncology Group Protocol 02-25. Available at: http://www.rtog.org/members/protocols/0225/0225.pdf. Accessed August 26, 2008.

[15] Radiation Therapy Oncology Group Protocol 06-15. Available at: http://www.rtog.org/members/protocols/0615/0615.pdf. Accessed August 26, 2008, 2008.

[16] Lin S, Pan J, Han L, et al. Nasopharyngeal carcinoma treated with reduced-volume intensity-modulated radiation therapy: report on the 3-year outcome of a prospective series [J]. International Journal of Radiation Oncology Biology Physics, 2009, 75 (4): 1071-1078.

[17] Yi J L, Gao L, Xu G Z, et al. Target volume delineation of IMRT for nasopharyngeal carcinoma-the experience of Cancer Hospital, Chinese Academy of Medical Sciences.[J]. Journal of Cancer Control and Treatment, 2011, 24 (3): 157-163.

[18] Committee of Chinese Clinical Staging of Nasopharyngeal Carcinoma. 2010 Consensus Guidelines for intensity-modulated radiotherapy target volume and dosimetric planning in nasopharyngeal carcinoma [J]. Chin J Radiat Oncol, 2011, 20 (4): 267-269.

[19] Lee A W, Ng W T, Pan J J, et al. International guideline for the delineation of the clinical target volumes (CTV) for nasopharyngeal carcinoma. [J]. Radiotherapy \& Oncology, 2017, 126 (1): 25-36. 